\title{
A low cost TDOA Localization System: Setup, Challenges and Results
}

\author{
Noha El Gemayel, Sebastian Koslowski, Friedrich K. Jondral \\ Communications Engineering Lab, Karlsruhe Institute of Technology (KIT), Germany \\ \{noha.gemayel, sebastian.koslowski,friedrich.jondral\}@kit.edu \\ and Joachim Tschan, LS Telcom, JTschan @LStelcom.com
}

\begin{abstract}
In this paper we present a prototype location estimation system which uses Time Difference of Arrival. The system consists of five low cost sensors that are synchronized using GPS time stamps. The sensors were installed on the rooftops of the university campus. The system is based on software defined radios which enable receiving signals with different carrier frequencies and sampling rates with the aim of applying it as a frequency monitoring and regulating system. The paper presents the expected challenges we face from the system and compares them to the measured results.
\end{abstract}

\section{INTRODUCTION}

Locating and tracking different signal sources is an increasingly interesting research topic. In modern technologies, the applicability of such information is huge. Different users are therefore looking into finding new methods that can accurately locate signal sources. This paper presents such a localization system using low cost hardware. Beside the mobile vendors that aim at applying the information in navigation, frequency regulators can use the information to localize unlicensed users as well as to dynamically allocate white spaces in the frequency, time and space domain. Depending on the use case, the position estimation techniques vary in their requirements and their accuracy. The positioning system we are presenting in this paper aims at providing the information passively and for different carrier frequencies, bandwidths and signals.

For this matter, Time Difference of Arrival (TDOA) was chosen because of its relatively high accuracy despite the fact that it does not require information about the transmitted signal. TDOA is mainly a two step method. In the first step, the time differences of arrivals of the signal of interest to the different sensors are measured. This can be done by crosscorrelation and peak detection of the sensors' signals. Many methods have been presented that deal with enhancing the correlation result, among them [1],[2]. In the second step, the measured TDOAs are used to solve the hyperbolic system. Closed form algorithms and iterative algorithms can be found in [3],[4]. Kalman Filters are also widely used because of their robustness and high accuracy [5],[6].

This paper is organized as follows. In section 2, the Time Difference of Arrival method is described. Section 3 presents the expected challenges of the system. Section 4 contains the setup of the test system, including the hardware of the positioning system and details of the transmitted signals. Section 5 contains some important results of our tested scenario.

\section{TIME DIFFERENCE OF ARRIVAL}

\section{A. TDOA Geometry}

The basic idea of Time Difference of Arrival is illustrated in Fig. 1. A TDOA measurement $\tau_{i, j}$ between two references $i$ and $j$ can be transformed into a distance difference $d_{i, j}$ :

$$
d_{i j}=d_{i}-d_{j}=c \cdot\left(t_{i}-t_{j}\right)=c \cdot \tau_{i, j}
$$

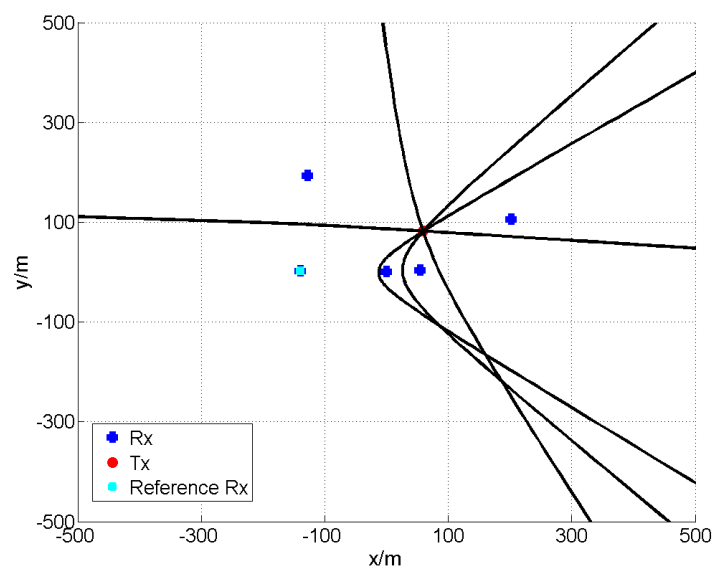

Fig. 1. TDOA-Geometry

Each distance difference can be described as a hyperbola of possible transmitter positions. The intersection of hyperbolas from multiple TDOAs yields the transmitter position.

Due to measurement errors, the hyperbolas shift and yield multiple intersection points. The position estimation aims at minimizing the square error. For zero mean errors, the least squares estimate would yield the optimal solution. In multipath channels, a Non Line of Sight (NLOS) propagation between the transmitter and the sensors can occur and would result in a biased TDOA estimation error. Many approaches were presented to solve this problem, either by identifying the NLOS sensors and eliminating them [7] or by weighting the TDOAs according to their reliability [8].

\section{B. Measurement of TDOA}

To be able to solve the hyperbolic equation system, the TDOAs must be obtained from the received signals. A TDOA system requires sensors are which are accurately synchronized 
among each other. Cross-correlating signals of a pair of sensors $\left(r_{i}, r_{j}\right)$ yields the requested TDOA by detecting the peak position. For an accurate estimation of the cross-correlation, the used time window per time difference estimation should be much larger than the maximum delay $T_{o}>>\tau_{\max }$.

$$
\begin{aligned}
C(\tau) & =\frac{1}{T_{o}} \int_{0}^{T_{o}} r_{i}(t) r_{j}(t+\tau) d t \\
\hat{\tau}_{i, j} & =\arg \max _{\tau}[C(\tau)]
\end{aligned}
$$

\section{System ChaLlenges}

\section{A. Discrete Signal Fractional Delay Estimation}

The previously mentioned cross-correlation was described in a continuous system (3). The signals we deal with are sampled with a frequency $F_{s}$, the correlator $C_{D}$ is estimated by

$$
C_{D}(n)=\frac{1}{N} \sum_{k=1}^{N} r_{i}\left(k T_{s}\right) r_{j}\left(k T_{s}+n T_{s}\right)
$$

with $T_{s}$ being the sampling interval. Take for example a sampling frequency of $10 \mathrm{MHz}$, the peak detection yields time differences with an accuracy of $T_{s}=\frac{1}{10 \mathrm{MHz}}=100 \mathrm{~ns}$ which in distance difference is an accuracy of $30 \mathrm{~m}$. This quantization effect can be described as noise with a variance of $\sigma_{q}^{2}=\frac{T_{s}^{2}}{12}$. To eliminate this effect, a quadratic interpolation is done. Assuming that the cross- correlation of the two signals can be expressed in terms of the autocorrelation of one of the signals and in the case of a band limited signal, the autocorrelation is symmetric around zero, which means for the cross-correlation a symmetry around TDOA. The function can then be approximated by a parabola of the following form [2].

$$
C_{D}(\tau)=a \tau^{2}+b \tau+c
$$

And the TDOA lies in the apex of the parabola:

$$
\hat{\tau}_{i, j}=-\frac{b}{2 a}
$$

Inserting the detected maximum of the discrete crosscorrelation and its two neighbouring samples in equation (5) and solving equation (6) yields the approximated continuous maximum of the cross- correlation.

\section{B. Low Pass Filter Response}

Another challenge we face in our system is the trade off between the width of the lowpass filter we use and the correlation accuracy. The low pass filter should filter out unwanted signals lying outside the interesting bandwidth. On the other hand, filtering all signals before cross-correlating them, the autocorrelation of the filter response would be visible in the cross-correlation of the signals. The smaller the bandwidth of the filter, the wider the correlation peak of the filter response and the wider the correlation peak of the filtered signals. Cross-correlating two filtered signals results in a lower accuracy of the estimated TDOA due to wider correlation peaks.

\section{Correlation Length and Update Rate}

A frequency monitoring system should be able to update the information about the different transmitted signals regularly. To do so, the incoming data is divided into sequences of $L$ samples and processed one after another. The update rate is $R_{u p} \approx \frac{F_{s}}{L} \frac{1}{\sec }$ without considering the computation time. On the other hand, the time delay estimation is highly dependant on the observation time. Assuming the simple case of an AWGN-channel with equal SNRs, and assuming a sufficient SNR, the Cramer Rao Lower Bound of the time difference estimate is given by [9]:

$$
\sigma_{\hat{\tau}}^{2}=\frac{3}{8 \pi^{2}}\left(\frac{1+2 \mathrm{SNR}}{\mathrm{SNR}^{2}}\right) \frac{1}{T_{o} B^{3}}
$$

The variance of the time delay estimate increases with lower bandwidths and lower SNRs. To compensate this, the only parameter to adjust at the receivers' side would be longer observation windows, resulting in lower update rates.

\section{Test Setup}

Our setup is comprised of five identical sensor stations installed on the rooftops of suitable campus buildings. Fig. 2 shows the chosen site on the southern part of the KIT campus. During the measurements these sensor stations record time-stamped IQ-Data which can be used for offline testing of different TDOA estimation and multilateration algorithms. Each sensor contains a Universal Software Radio Peripheral (USRP) 2 with a WBX daughterboard [10]. This enables us to run tests in various spectral bands $(50 \mathrm{MHz}-2.2 \mathrm{GHz})$ using a wide range of sample rates. The USRP is connected to and controlled by a standard industrial PC equipped with large and fast storage space.

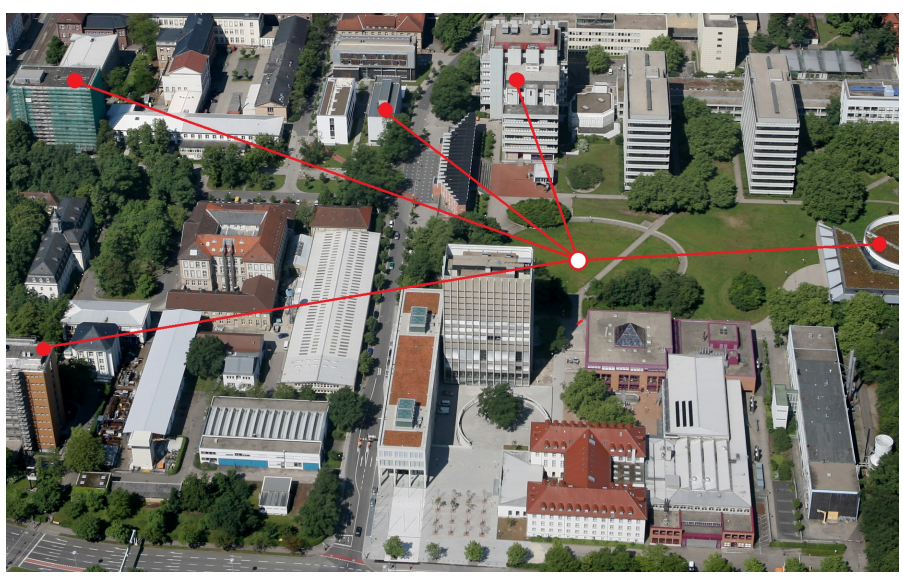

Fig. 2. Test Setup on Campus

The crucial part of every TDOA-based measurement system is tagging the recorded samples with their respective reception times. Our sensor stations each have a built-in GPS receiver (Garmin GPS16-HVS,[11]) which provides a highly accurate time and is also used to discipline the USRPs oscillators. Using the PPS pulses generated by the GPS receivers and the 
mechanisms provided by the USRPs drivers [12], each packet of samples sent to the PC contains the exact reception time in its header.

To get well defined and reproducable test signals for our measurements, the transmitter is comprised of yet another USRP2 with a WBX daughterboard. Random, linearly modulated data with various symbol rates are used as transmit signals. To verify the accuracy of our sensor stations and their timing information the output of the transmitting USRP is additionally amplified. This way even the more distant sensor stations get comfortably high signal strengths.

The distances between the transmitter and the sensors vary between $80 \mathrm{~m}$ and $230 \mathrm{~m}$. Four of the five used sensors are expected to have Line of Sight (LOS) to the transmitter, one sensor has NLOS.

\section{RESULts}

The scenario we are presenting in this paper consists of a transmitted pseudo random signal. The signal bandwidth is $1 \mathrm{MHz}$ and the used carrier frequency is $431 \mathrm{MHz}$. The sensors are set to a sampling frequency of $5 \mathrm{MHz}$. Fig. 3 shows the spectrum of the received signals at the five sensors. Sensor 3 has NLOS, which can be seen in the obvious fading effect. In addition to that, the signal powers vary according to the distances.

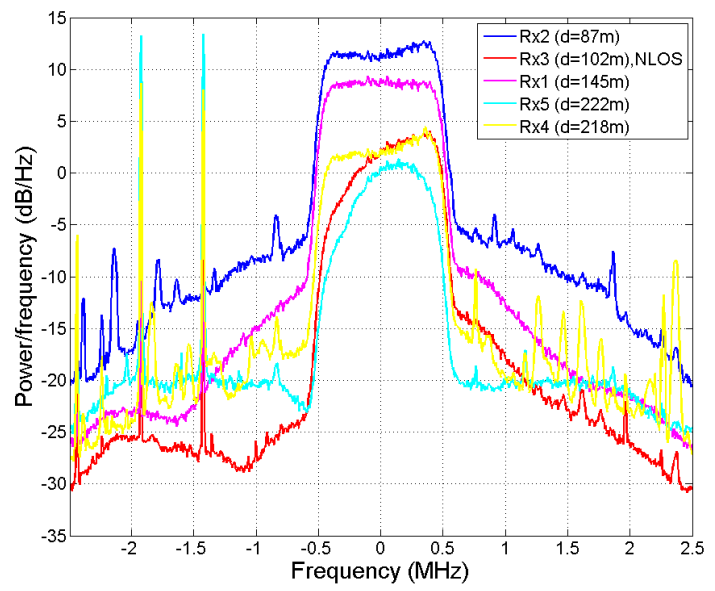

Fig. 3. Spectrum of the Received Signals

The recorded and time stamped IQ-Data were gathered from the sensors and processed using Matlab. The first step consisted of filtering the interesting bandwidth. Afterwards the data was synchronized on a sample basis. The synchronized data was cross-correlated and interpolated around the detected peak. For the position estimation, a number of algorithms were implemented. While [3] presents an iterative algorithm that linearizes the TDOA equation system, [4] presents a closed form algorithm. [8] and [7] deal with NLOS error mitigation. [5] and [6] present two Kalman Filters that can handle nonlinear systems. The final TDOA estimate was fed to one of the implemented localization algorithms.
Fig. 4 shows an example of a cross-correlation between recorded signals of two sensors. The cross-correlation of an ideal low pass filter is shown for comparison. The degradation due to various channel effects and due to the non ideal filter that we used can be seen. The correlation coefficient at the peak is not one, side lobes are larger and distortion can be seen. The figure zooms in around the correlation peak to show the interpolation using the three discrete values around the peak. The interpolation is still an approximation of the original function, the bias due to interpolation was given in [13]. It depends on the window used for correlation as well as the position of the peak between the discrete values.

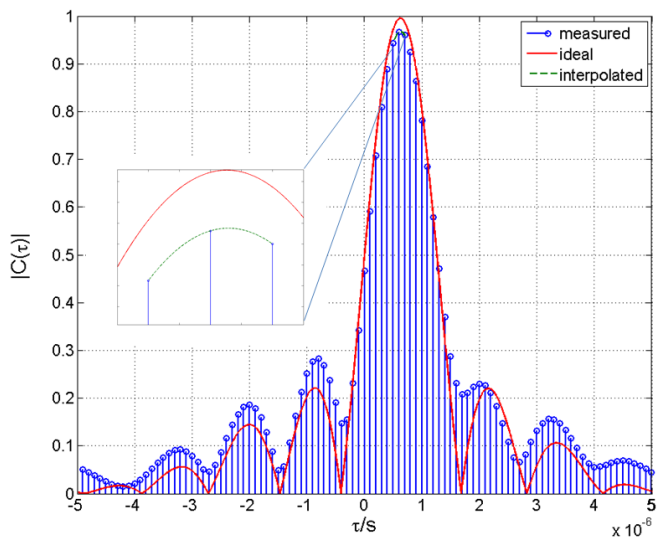

Fig. 4. Cross-Correlation and Interpolation

Fig. 5 shows two histograms of the distance difference $\left(d_{i, j}\right)$ error in meters. The results show the estimates of two sensors, one with LOS and one with NLOS, referenced to a LOS sensor. Both histograms show a bias of the distance difference estimate. While the LOS sensor bias is around $12 \mathrm{~m}$, the NLOS sensor bias is $45 \mathrm{~m}$. The bias of the LOS sensor is due to synchronization errors as well as interpolation errors. The histograms also show different standard deviations of the estimates. While the LOS sensor has a standard deviation of $12 \mathrm{~m}$, the NLOS sensor has one of $36 \mathrm{~m}$. This proves the assumption made by [8] and [7] that NLOS sensors deliver TDOA estimates with higher standard deviations. The corresponding Gaussian distributions are plotted for comparison. Many papers in the literature model the TDOA error as Gaussian noise, which proves to be a very limited assumption.

Fig. 6 shows the position estimate using 20 seconds of the signals and correlating each 0.01 seconds, 2000 position estimates were calculated using two algorithms. The results of using the Taylor Series Estimation (TSE) presented in [3] are plotted in black. The root mean square error of the estimates is about $23 \mathrm{~m}$. The results using the Extended Kalman Filter presented in [5] are plotted using the color bar to show the filter steps. The position error after 2000 steps is $10 \mathrm{~m}$.

To give an idea of how the different variables affect the result, table I shows the results using three different filter bandwidths (BW) and three different observation windows 


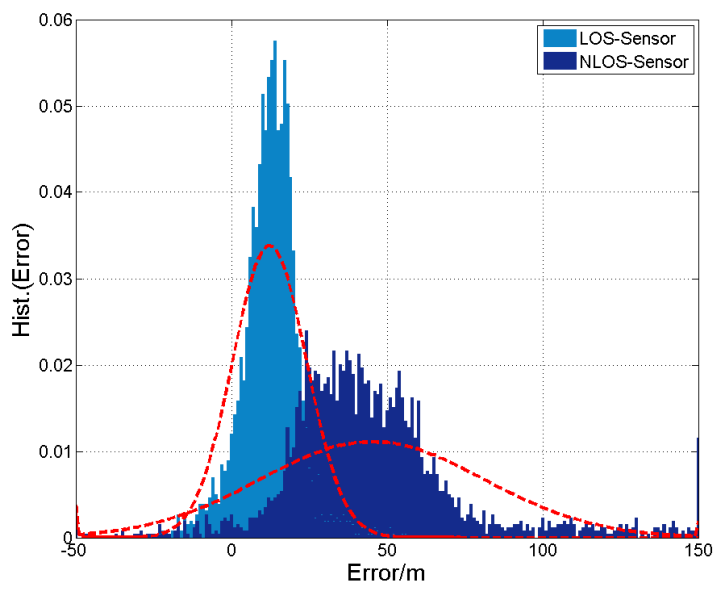

Fig. 5. Histogram of Distance Difference Errors

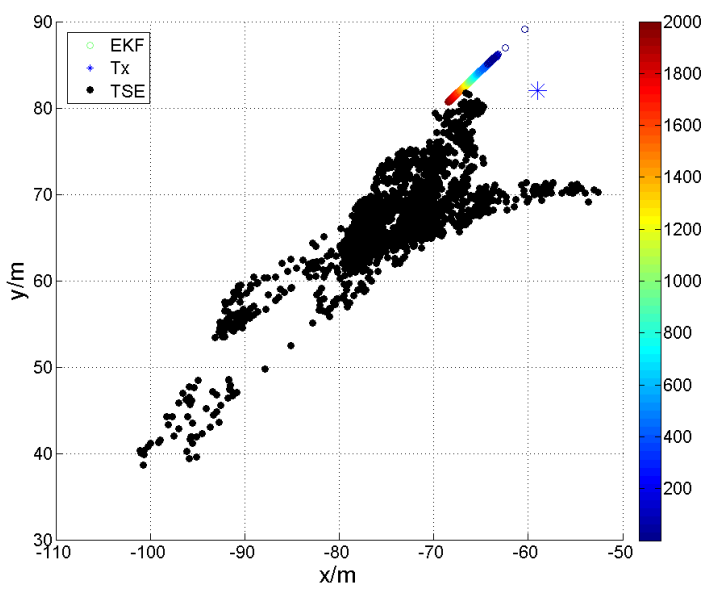

Fig. 6. Position Estimate using the EKF and the TSE Algorithms

$\left(T_{o}\right)$. The table shows the standard deviation of the four distance difference estimates we get using one of the sensors as reference sensor. The results show a small degradation due to a smaller bandwidth for all except $d_{3,1}$ which includes the NLOS sensor, where a smaller bandwidth leads to more accurate results. This can be explained by looking into Fig. 3: the smaller the bandwidth, the whiter the signal and the less fading effects we observe.

Increasing the observation window shows a saturation effect, especially between the $1 \mathrm{~ms}$ and $10 \mathrm{~ms}$ cases. The improvement due to a larger averaging window reaches its best. The remaining error sources can not be eliminated by longer windows like the signal noise can. Fading effects and synchronization errors must be handled differently. In that case, applying more Kalman Filter steps instead of correlating over a larger window would be of more use for the position estimation accuracy.

\begin{tabular}{|c|c|c|c|c|c|}
\hline BW & $\mathrm{T}_{o}$ & \multicolumn{4}{|c|}{ stdv. $\left(d_{i, j}\right) / \mathrm{m}$} \\
\hline & & $\mathrm{d}_{2,1}$ & $\mathrm{~d}_{3,1}$ & $\mathrm{~d}_{4,1}$ & $\mathrm{~d}_{5,1}$ \\
\hline $250 \mathrm{kHz}$ & $100 \mu s$ & 12 & 25 & 17 & 15 \\
\hline $250 \mathrm{kHz}$ & $1 \mathrm{~ms}$ & 6 & 24 & 12 & 10 \\
\hline $250 \mathrm{kHz}$ & $10 \mathrm{~ms}$ & 6 & 24 & 12 & 10 \\
\hline $500 \mathrm{kHz}$ & $100 \mu s$ & 7 & 25 & 12 & 10 \\
\hline $500 \mathrm{kHz}$ & $1 \mathrm{~ms}$ & 6 & 25 & 12 & 9 \\
\hline $500 \mathrm{kHz}$ & $10 \mathrm{~ms}$ & 6 & 25 & 12 & 9 \\
\hline $1 \mathrm{MHz}$ & $100 \mu s$ & 5 & 27 & 11 & 8 \\
\hline $1 \mathrm{MHz}$ & $1 \mathrm{~ms}$ & 5 & 26 & 10 & 8 \\
\hline $1 \mathrm{MHz}$ & $10 \mathrm{~ms}$ & 5 & 26 & 10 & 8 \\
\hline
\end{tabular}

TABLE I

RESULTS OF VARYING FILTER BANDWIDTH AND OBSERVATION WINDOW

\section{CONClusion AND Future Work}

In this paper we showed a test setup for a TDOA geolocation system using five sensors that were placed on the rooftops of the university campus. The goal of the paper was to present the expected challenges we face from the system and to verify the implemented algorithms. The results proved the functionality of the hardware. The time stamped IQ-Data were cross-correlated to obtain Time Differences of Arrivals. The implemented algorithms delivered accurate results of the position estimate. In future work, we analyze the channel effects more precisely using a new data set and comparing them to simulated results.

\section{REFERENCES}

[1] C. Knapp and G. Carter, "The generalized correlation method for estimation of time delay," IEEE Transactions on Acoustics, Speech and Signal Processing, vol. 24, no. 4, pp. 320 - 327, Aug. 1976.

[2] G. Jacovitti and G. Scarano, "Discrete time techniques for time delay estimation," IEEE Transactions on Signal Processing, vol. 41, no. 2, pp. $525-533$, Feb. 1993.

[3] W. Foy, "Position-location solutions by Taylor-series estimation," IEEE Transactions on Aerospace and Electronic Systems, vol. AES-12, no. 2, pp. $187-194$, March 1976.

[4] Y. Chan and K. Ho, "A simple and efficient estimator for hyperbolic location," IEEE Transactions on Signal Processing, vol. 42, no. 8, pp. 1905 -1915, Aug. 1994.

[5] C. Hongyang, D. Ping, X. Yongjun, and L. Xiaowei, "A robust location algorithm with biased extended Kalman filtering of tdoa data for wireless sensor networks," in International Conference on Wireless Communications, Networking and Mobile Computing, 2005. Proceedings. 2005, vol. 2, Sept. 2005, pp. $883-886$.

[6] C. Savage, R. Cramer, and H. Schmitt, "TDOA geolocation with the unscented Kalman filter," in Proceedings of the 2006 IEEE International Conference on Networking, Sensing and Control, 2006. ICNSC '06., 0-0 2006, pp. $602-606$.

[7] L. Cong and W. Zhuang, "Non-line-of-sight error mitigation in TDOA mobile location," in Global Telecommunications Conference, 2001. GLOBECOM '01. IEEE, vol. 1, 2001, pp. 680 -684 vol.1.

[8] P.-C. Chen, "A non-line-of-sight error mitigation algorithm in location estimation," in Wireless Communications and Networking Conference, 1999. WCNC. 1999 IEEE, 1999, pp. 316 -320 vol.1.

[9] G. Carter, "Coherence and time delay estimation," Proceedings of the IEEE, vol. 75, no. 2, pp. 236 - 255, Feb. 1987.

[10] "Ettus research." [Online]. Available: https://www.ettus.com/

[11] "Garmin GPS 16 Specifications," http://www8.garmin.com/products/gps16/spec.

[12] "Usrp hard driver," http://files.ettus.com/uhd_docs/manual/.

[13] R. Boucher and J. Hassab, "Analysis of discrete implementation of generalized cross correlator," IEEE Transactions on Acoustics, Speech and Signal Processing, vol. 29, no. 3, pp. 609 - 611, Jun. 1981. 